\title{
Hadamard product of analytic functions and some special regions and curves
}

Krzysztof Piejko and Janusz Sokół*

Dedicated to Professor Hari M Srivastava

"Correspondence: jsokol@prz.edu.pl Department of Mathematics, Rzeszów University of Technology, Al. Powstańcó Warszawy 12, Rzeszów, 35-959, Poland

\begin{abstract}
In this paper we present some new applications of convolution and subordination in geometric function theory. The paper deals with several ideas and techniques used in this topic. Besides being an application to those results, it provides interesting corollaries concerning special functions, regions and curves.

MSC: Primary 30C45; secondary 30C80
\end{abstract}

Keywords: Hadamard product; univalent functions; convex functions; closed convex hull; convolution; subordination

\section{Introduction}

By $\mathcal{H}$ we denote a class of analytic functions in the unit disc $\Delta=\{z \in \mathbb{C}:|z|<1\}$. Let $\mathcal{K}$ denote the subclass of $\mathcal{H}$ consisting of functions normalized by $f(0)=0, f^{\prime}(0)=1$ and satisfying

$$
\mathfrak{R e}\left(1+\frac{z f^{\prime \prime}(z)}{f^{\prime}(z)}\right)>0 \quad \text { for } z \in \Delta .
$$

Of course the functions from $\mathcal{K}$ map the unit disc onto convex domains.

We say that the function $f \in \mathcal{H}$ is subordinate to the function $g \in \mathcal{H}$ in the unit disc $\Delta$ (written $f \prec g$ ) if and only if there exists an analytic function $w \in \mathcal{H}$ such that $w(0)=0$, $|w(z)|<1$ and $f(z)=g(w(z))$ for $z \in \Delta$. Therefore $f \prec g$ in $\Delta$ implies $f(\Delta) \subset g(\Delta)$. If, additionally, $g$ is univalent in $\Delta$, then

$$
f \prec g \text { if and only if } f(0)=g(0) \text { and } f(\Delta) \subset g(\Delta) \text {. }
$$

Recall that the Hadamard product or convolution of two power series,

$$
f(z)=\sum_{n=0}^{\infty} a_{n} z^{n} \quad \text { and } \quad g(z)=\sum_{n=0}^{\infty} b_{n} z^{n}
$$

is defined as

$$
(f * g)(z)=f(z) * g(z)=\sum_{n=0}^{\infty} a_{n} b_{n} z^{n} .
$$

(0) 2013 Piejko and Sokół; licensee Springer. This is an Open Access article distributed under the terms of the Creative Commons Attribution License (http://creativecommons.org/licenses/by/2.0), which permits unrestricted use, distribution, and reproduction in any medium, provided the original work is properly cited. 
The convolution has the algebraic properties of ordinary multiplication. We now look at some problems on convolution and at some of the relations between the convolution and the subordination. One can consider the following problems.

Problem 1 Find the subclasses $\mathcal{A}, \mathcal{B}, \mathcal{C}$ of the class $\mathcal{H}$ such that

$$
\mathcal{A} * \mathcal{B}=\mathcal{C}
$$

where

$$
\mathcal{A} * \mathcal{B}=\{h(z)=(f * g)(z) \text { for all } f \in \mathcal{A} \text { and for all } f \in \mathcal{B}\} .
$$

Problem 2 Let the sets $M \subset \mathbb{C}$ and $N \subset \mathbb{C}$ be given. Find a set $K \subset \mathbb{C}$, as small as possible, such that

$$
f(\Delta) \subset M \quad \text { and } \quad g(\Delta) \subset N \quad \Rightarrow \quad(f * g)(\Delta) \subset K \quad \text { for all } f, g \in \mathcal{D} \text {, }
$$

where $\mathcal{D}$ is some subclass of $\mathcal{H}$.

If $M=F(\Delta)$ and $N=G(\Delta)$, where $F, G \in \mathcal{H}$ are univalent in $\Delta$, then, by (1.2), Problem 2 above becomes the following one:

Problem 3 Let the univalent functions $F, G$ be given. Find a function $H, H(\Delta)=K$, and a class $\mathcal{D} \subset \mathcal{H}$ such that

$$
f \prec F \quad \text { and } g \prec G \Rightarrow f * g \prec H \quad \text { for all } f, g \in \mathcal{D} \text {. }
$$

Ruscheweyh and Sheil-Small [1] proved the Pòlya-Schoenberg conjecture that the class of convex univalent functions is preserved under convolution, namely $\mathcal{K} * \mathcal{K}=\mathcal{K}$. They proved also that the class of starlike functions and the class of close-to-convex functions are closed under convolution with the class $\mathcal{K}$. Another solution of Problem 1 is the following theorem.

Theorem A (see [2]) Let us denote

$$
\mathcal{P}_{x}=\{p \in \mathcal{H}: p(0)=1 \text { and } \mathfrak{R} e\{p(z)\}>x\} .
$$

Suppose that $\alpha \leq 1, \beta \leq 1$. Then

$$
\mathcal{P}_{\alpha} * \mathcal{P}_{\beta}=\mathcal{P}_{\gamma},
$$

where $\gamma=1-2(1-\alpha)(1-\beta)$.

Problem 2, when the sets $M$ and $K$ are discs $\mathfrak{K}\left(s_{i}, R_{i}\right)$ with center $s_{i}$ and radius $R_{i}$, was considered in [3] and in other papers. One of results obtained there is the following theorem. 
Theorem B (see [3]) Let $s_{i} \in \mathbb{C}, R_{i} \in \mathbb{R} ; i=1$, 2. If $f, g \in \mathcal{H}$ and $f(0)=a, g(0)=b$, then the following implication holds:

$$
f(\Delta) \subset \mathfrak{K}\left(s_{1}, R_{1}\right) \quad \text { and } \quad g(\Delta) \subset \mathfrak{K}\left(s_{2}, R_{2}\right) \quad \Rightarrow \quad(f * g)(\Delta) \subset \mathfrak{K}(s, R) \quad \text { for all } f, g \in \mathcal{H} \text {, }
$$

where

$$
s=a b+m\left(s_{1}-a\right)\left(s_{2}-b\right), \quad R=m R_{1} R_{2}, \quad m=\frac{\left(R_{1}^{2}-\left|s_{1}-a\right|^{2}\right)\left(R_{2}^{2}-\left|s_{2}-a\right|^{2}\right)}{R_{1}^{2} R_{2}^{2}-\left|s_{1}-a\right|^{2}\left|s_{2}-a\right|^{2}} .
$$

In this paper we shall look for a solution of the following modified Problem 3.

Problem 4 Find a function $F \in \mathcal{H}$ and a class $\mathcal{D} \subset \mathcal{H}$ such that

$$
g \prec f \quad \Rightarrow \quad g * F \prec f * F \quad \text { for all } f \in \mathcal{D}, g \in \mathcal{H} \text {. }
$$

The next theorem is a solution of Problem 4.

Theorem C (see [4]) Assume that $f \in \mathcal{H}, f^{\prime}(0) \neq 0$, and that it satisfies

$$
\mathfrak{R e}\left\{1+\frac{z f^{\prime \prime}(z)}{f^{\prime}(z)}\right\}>-\frac{1}{2} \quad \text { for } z \in \Delta
$$

If $g \prec f$, then

$$
L[g] \prec L[f]
$$

where

$$
L[f](z)=\frac{2}{z} \int_{0}^{z} f(t) \mathrm{d} t
$$

is the Libera operator [5].

$$
\begin{aligned}
& \text { If } f(z)=a_{0}+a_{1} z+a_{2} z^{2}+a_{3} z^{3}+\cdots, \text { then we have } \\
& \qquad \begin{aligned}
L[f](z) & =\frac{2}{z} \int_{0}^{z} f(t) \mathrm{d} t=\sum_{n=0}^{\infty} \frac{2 a_{n}}{n+1} z^{n} \\
& =f(z) *\left(\frac{2}{z} \log \frac{1}{1-z}\right) .
\end{aligned}
\end{aligned}
$$

Therefore, Theorem C may be written as

$$
\begin{gathered}
g \prec f \Longrightarrow g *\left(\frac{2}{z} \log \frac{1}{1-z}\right) \prec f *\left(\frac{2}{z} \log \frac{1}{1-z}\right) \\
\text { for all } f \in \mathcal{K}(-1 / 2), g \in \mathcal{H},
\end{gathered}
$$

where

$$
\mathcal{K}(\alpha):=\left\{f \in \mathcal{H}: f^{\prime}(0) \neq 0 \text { and } \Re \mathfrak{R}\left\{1+\frac{z f^{\prime \prime}(z)}{f^{\prime}(z)}\right\}>\alpha \text { for } z \in \Delta\right\} .
$$


Theorem $\mathrm{C}$ improves an earlier result from [6] with the stronger assumption $f \in \mathcal{K}(0)$ and the same conclusion. Moreover, if we assume more, that $f \in \mathcal{K}(0)$ and $f$ is univalent in $\Delta$, then in (1.3) instead of $F(z)=-2 / z \log (1-z)$ we can put any convex univalent function. This is contained in the following result due to Ruscheweyh and Stankiewicz [7].

Theorem D (see [7]) Let $F$ and $G$ be convex univalent in $\Delta$. Then, for all functions $f, g \in \mathcal{H}$,

$$
f \prec F \quad \text { and } \quad g \prec G \quad \Longrightarrow \quad f * g \prec F * G \text {. }
$$

This relationship allows us to obtain several subordination results about convolution. Note that there are no assumptions about the normalization of functions. It is one of the solutions of Problem 3. Many of the convolution properties were studied by Ruscheweyh in [8], and they have found many applications in various fields. The book [8] is also an excellent survey of the results. For the recent results on the Hadamard product in geometric function theory, see [9-13].

\section{A family of functions}

Throughout this section we consider the family of analytic functions

$$
F_{s}(z)=\frac{z}{1-s z^{2}} \quad(z \in \Delta)
$$

where $s \in[0,1]$. In Section 3 we prove that for given $s \in[0,1)$ the curve $F_{s}\left(e^{i \varphi}\right), \varphi \in[0,2 \pi)$ is the Booth lemniscate. Let $\mathcal{S}^{*}$ denote a class of starlike functions consisting of functions $f$ such that $z f^{\prime} \in \mathcal{K}$.

Lemma 2.1 The function $F_{s}$ given by (2.1) is a starlike univalent function for $s \in[0,1]$.

Proof We have

$$
\mathfrak{R e}\left\{\frac{z F_{s}^{\prime}(z)}{F_{s}(z)}\right\}=\mathfrak{R e}\left\{\frac{1+s z^{2}}{1-s z^{2}}\right\}>0 \quad \text { for all } z \in \Delta .
$$

Thus, it is obvious that $F_{s}$ is a starlike univalent function for $s \in[0,1]$.

Lemma 2.2 Let the function $F_{s}$ be of the form (2.1), and let $\mathcal{K}(-1 / 2)$ be given by (1.4). If $|s| \leq 6-\sqrt{33} \approx 0.255437$, then $F_{s} \in \mathcal{K}(-1 / 2)$.

Proof Assume that $s z^{2}=r(\cos t+i \sin t), 0 \leq r<1,0 \leq t \leq 2 \pi$ and set

$$
H(t, r):=\frac{z F_{s}^{\prime \prime}(z)}{F_{s}^{\prime}(z)}+2=\frac{6\left(1+s z^{2}\right)-4}{1-\left(s z^{2}\right)^{2}}=\frac{2+6 r \cos t+6 i r \sin t}{1-r^{2} \cos 2 t-i r^{2} \sin 2 t} .
$$

By (1.4) if $\mathfrak{R e}\{H(t, r)\}>1 / 2$ for all $r \in\left[0, r_{0}\right), t \in[0,2 \pi]$, then $F_{s} \in \mathcal{K}(-1 / 2)$ for all $|s| \leq r_{0}$. After some calculations, we obtain

$$
\mathfrak{R e}\{H(t, r)\}=\frac{-4 r^{2} x^{2}+6 r\left(1-r^{2}\right) x+2\left(1+r^{2}\right)}{\left(1+r^{2}\right)^{2}-4 r^{2} x^{2}}:=\frac{n(x, r)}{d(x, r)},
$$


where $x=\cos t$. Thus we shall find $r_{0}$ in $[0,1]$ such that

$$
\frac{n(x, r)}{d(x, r)}>1 / 2 \quad \text { for all } r \in\left[0, r_{0}\right) \text { and for all } x \in[-1,1]
$$

It is easy to verify that $d(x, r)>0$ for all $r \in[0,1)$ and for all $x \in[-1,1]$, and that $n(-1, r)=$ $2\left(1-r^{2}\right)(1-3 r) \leq 0$ for all $r \in[1 / 3,1)$. Hence, to find $r_{0}$ in $[0,1]$ satisfying $(2.4)$, we may restrict our considerations to the interval $[0,1 / 3)$. Then, analyzing $n(x, r)$ as a function of one variable $x$, we get

$$
\min \{n(x, r):-1 \leq x \leq 1\}=n(-1, r)=2\left(1-r^{2}\right)(1-3 r)>0
$$

for all $r \in[0,1 / 3)$. Moreover, we see that

$$
\min \{d(x, r):-1 \leq x \leq 1\}=d(-1, r)=\left(1-r^{2}\right)^{2}>0 .
$$

Therefore, if $0 \leq r<1 / 3$, then

$$
\min \left\{\frac{n(x, r)}{d(x, r)}: x \in[-1,1]\right\}=\frac{2(1-3 r)}{1-r^{2}} .
$$

To find $r_{0}$ in $[0,1 / 3)$ satisfying (2.4), it is sufficient to solve the inequality

$$
\frac{2(1-3 r)}{1-r^{2}}>\frac{1}{2}
$$

which is true for $0 \leq r \leq r_{0}=6-\sqrt{33}$.

Moreover, analogous consideration as in the above proof leads us to the fact that $F_{s} \notin$ $\mathcal{K}(-1 / 2)$ for $s>6-\sqrt{33} \approx 0.255437$. In Lemma 3.2 we show that $F_{s} \in \mathcal{K}$ for $0 \leq s \leq 3-$ $2 \sqrt{2} \approx 0.1715$.

Theorem 2.3 If $0 \leq s \leq 6-\sqrt{33} \approx 0.255437$ and $f \in \mathcal{H}$, then

$$
f \prec F_{s} \quad \Longrightarrow \quad L[f] \prec L\left[F_{s}\right]
$$

where

$$
L[f](z)=\frac{2}{z} \int_{0}^{z} f(t) \mathrm{d} t
$$

is the Libera operator.

Proof By Lemma 2.2 we obtain

$$
\mathfrak{R e}\left\{1+\frac{z F_{s}^{\prime \prime}(z)}{F_{s}^{\prime}(z)}\right\}>-\frac{1}{2} \quad \text { for } z \in \Delta .
$$

Therefore, using Theorem C, we get (2.5). 
Corollary 2.4 If $0 \leq s \leq 6-\sqrt{33}$ and

$$
f(z)=\sum_{n=1}^{\infty} a_{n} z^{n}
$$

then

$$
\sum_{n=1}^{\infty} a_{n} z^{n} \prec \sum_{n=1}^{\infty} s^{n-1} z^{2 n-1} \quad \Longrightarrow \quad \sum_{n=1}^{\infty} \frac{a_{n}}{n+1} z^{n} \prec \sum_{n=1}^{\infty} \frac{s^{n-1}}{n+1} z^{2 n-1} .
$$

It is known [14] that if

$$
\sum_{n=0}^{\infty} \alpha_{n} z^{n} \prec \sum_{n=0}^{\infty} \beta_{n} z^{n}
$$

then

$$
\sum_{n=0}^{k}\left|\alpha_{n}\right|^{2} \leq \sum_{n=0}^{k}\left|\beta_{n}\right|^{2}
$$

Hence, for $0 \leq s \leq 6-\sqrt{33}$, Corollary 2.4 gives also that

$$
\begin{aligned}
\sum_{n=1}^{\infty} a_{n} z^{n} \prec F_{s}(z) \Rightarrow \sum_{n=1}^{\infty} \frac{\left|a_{n}\right|^{2}}{(n+1)^{2}} & \leq \sum_{n=1}^{\infty} \frac{s^{2(n-1)}}{(n+1)^{2}}=\frac{-1}{s^{2}}+\frac{1}{s^{2}} \int_{0}^{\infty} \frac{t}{e^{t}-s^{2}} \mathrm{~d} t \\
& =\frac{\Phi\left(s^{2}, 2,1\right)-1}{s^{2}},
\end{aligned}
$$

because for $\mathfrak{R e}\{a\}>0,-1 \leq x<1, \mathfrak{R e}\{c\}>0$,

$$
\Phi(x, c, a)=\sum_{k=0}^{\infty} \frac{x^{k}}{(k+a)^{c}}=\frac{1}{\Gamma(c)} \int_{0}^{\infty} \frac{t^{c-1} e^{-a t}}{1-x e^{-t}} \mathrm{~d} t
$$

Theorem 2.5 If $0 \leq s_{i} \leq 6-\sqrt{33}, i=1,2$, and the functions $g_{1}$ and $g_{2}$ are in the class $\mathcal{H}$, then

$$
g_{1} \prec F_{s_{1}} \quad \text { and } \quad g_{2} \prec F_{s_{2}} \quad \Longrightarrow \quad L\left[g_{1}\right] * L\left[g_{2}\right] \prec L\left[L\left[F_{s_{1} s_{2}}\right]\right]
$$

Proof By Theorem 2.3 we have $L\left[g_{1}\right] \prec L\left[F_{s_{1}}\right]$ and $L\left[g_{2}\right] \prec L\left[F_{s_{2}}\right]$. By Lemma $2.1 F_{s} \in \mathcal{S}^{*}$, $s \in[0,1]$, so the functions $L\left[F_{s_{1}}\right], L\left[F_{s_{2}}\right]$ are in $\mathcal{K}$ because $L\left[\mathcal{S}^{*}\right]=\mathcal{K}$. Using Theorem $\mathrm{D}$, we obtain

$$
L\left[g_{1}\right] * L\left[g_{2}\right] \prec L\left[F_{s_{1}}\right] * L\left[F_{s_{2}}\right]
$$

but $L\left[F_{s_{1}}\right] * L\left[F_{s_{2}}\right]=L\left[L\left[F_{s_{1} s_{2}}\right]\right]$ because

$$
F_{s_{1}}(z) * F_{s_{2}}(z)=\frac{z}{1-s_{1} z^{2}} * \frac{z}{1-s_{2} z^{2}}=\frac{z}{1-s_{1} s_{2} z^{2}}=F_{s_{1} s_{2}}(z) .
$$




\section{A family of sets}

In this section we investigate the properties of a one-parameter family of the sets $D(s)$, $s \in[0,1]$, such that

$$
D(s)=\left\{x+i y \in \mathbb{C}:\left(x^{2}+y^{2}\right)^{2}-\frac{x^{2}}{(1-s)^{2}}-\frac{y^{2}}{(1+s)^{2}}<0\right\},
$$

when $s \in[0,1)$ and

$$
D(1)=\{x+i y \in \mathbb{C}: x+i y \neq i t \text { for all } t \in(-\infty,-1) \cup(1, \infty)\}
$$

Lemma 3.1 Let $s \in[0,1]$, then $D(s)=F_{s}(\Delta)$, where

$$
F_{s}(z)=\frac{z}{1-s z^{2}} \quad(z \in \Delta)
$$

Proof The function $F_{s}$ is analytic in the unit disc, so for the proof we need to find an image of the circle $|z|=1$ under the function $F_{s}$. For $s \in[0,1)$ and for $\varphi \in[0,2 \pi)$, we have

$$
\begin{aligned}
F_{S}\left(e^{i \varphi}\right) & =\frac{e^{i \varphi}}{1-s e^{2 i \varphi}} \cdot \frac{1-s e^{-2 i \varphi}}{1-s e^{-2 i \varphi}}=\frac{e^{i \varphi}-s e^{-i \varphi}}{1+s^{2}-s\left(e^{2 i \varphi}+e^{-2 i \varphi}\right)} \\
& =\frac{(1-s) \cos \varphi+i(1+s) \sin \varphi}{1+s^{2}-2 s \cos 2 \varphi} .
\end{aligned}
$$

Let us define

$$
x=\mathfrak{R e}\left\{F_{s}\left(e^{i \varphi}\right)\right\}=\frac{(1-s) \cos \varphi}{1+s^{2}-2 s \cos 2 \varphi}
$$

and

$$
y=\mathfrak{I m}\left\{F_{s}\left(e^{i \varphi}\right)\right\}=\frac{(1+s) \sin \varphi}{1+s^{2}-2 s \cos 2 \varphi} .
$$

Then, after some calculations, we can obtain from (3.4) and (3.5) that

$$
x^{2}+y^{2}=\frac{1}{1+s^{2}-2 s \cos 2 \varphi} \quad \text { and } \quad(x, y) \neq(0,0) \text {. }
$$

Therefore, using the relations (3.3)-(3.5), we can find that $F_{s}\left(e^{i \varphi}\right)$ is an algebraic curve of order four whose equation in orthogonal Cartesian coordinates is

$$
\left(x^{2}+y^{2}\right)^{2}-\frac{x^{2}}{(1-s)^{2}}-\frac{y^{2}}{(1+s)^{2}}=0 \quad \text { and } \quad(x, y) \neq(0,0) \text {. }
$$

Thus, because $F_{s}(0)=0$ for $s \in[0,1)$, the set $F_{s}(\Delta)$ is bounded by the curve (3.7). Moreover, for $s=1$, the function $F_{s}$ becomes

$$
F_{1}(z)=\frac{z}{1-z^{2}}
$$

and it is easy to see that $F_{1}(\Delta)=D(1)$, see Figure 1 . Then the proof is completed. 


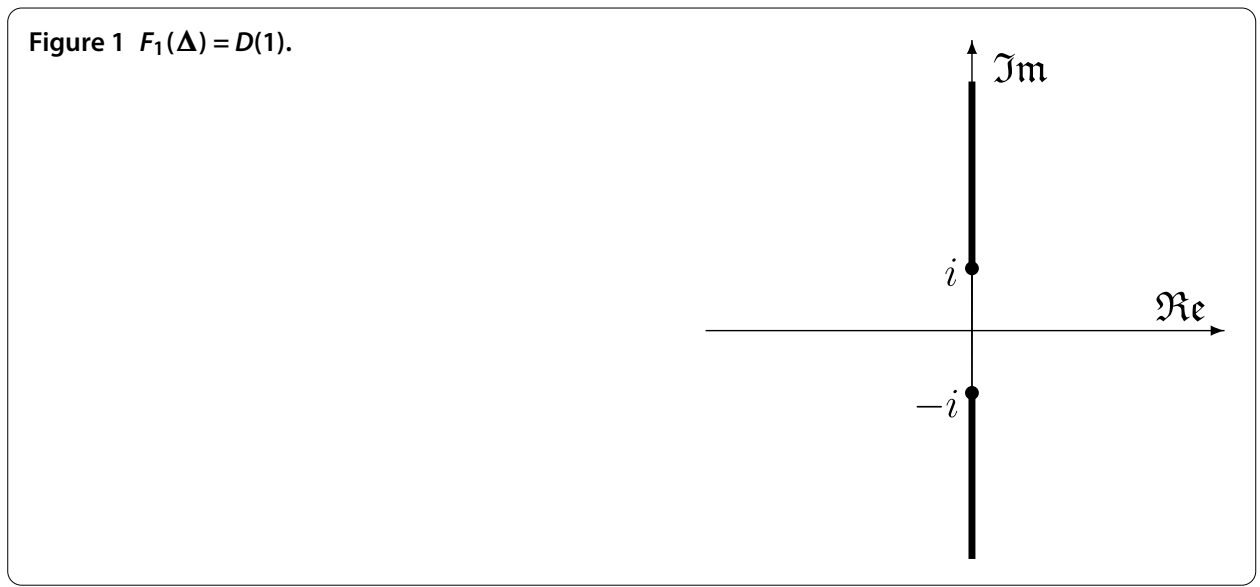

Notice that a curve described by

$$
\left(x^{2}+y^{2}\right)^{2}-\left(n^{4}+2 m^{2}\right) x^{2}-\left(n^{4}-2 m^{2}\right) y^{2}=0 \quad \text { and } \quad(x, y) \neq(0,0)
$$

is called the Booth lemniscate, named after Booth $[15,16]$. The Booth lemniscate is called elliptic if $n^{4}>2 m^{2}$, while for $n^{4}<2 m^{2}$ it is termed hyperbolic. Thus it is clear that the curve (3.7) is the Booth lemniscate of elliptic type. The Booth lemniscate is a special case of a Persian curve. The Booth lemniscate of elliptic type can be described geometrically in the following equivalent ways.

1. Suppose that $s \in[0,1)$. Let $\mathfrak{C}(S, R)$ be a circle with the center $S$ and the length of the radius $R$ such that

$$
S=\left(\frac{\sqrt{s}}{1-s^{2}}, 0\right) \quad \text { and } \quad R=\frac{1}{2(1-s)}
$$

A ray is drawn from the point $x$ on the circle $\mathfrak{C}(S, R)$ through the origin $o$ that cuts also the circle $\mathfrak{C}(S, R)$ at the point $z$. A point $v$ is also on the ray and satisfies

$$
|o v|=|x z| .
$$

If the point $x$ goes along the circle $\mathfrak{C}(S, R)$, then the point $v$ describes the curve (3.7), see Figure 2.

2. Let $s \in[0,1)$. Then the curve (3.7) consists of points $M$ such that

$$
\left|F_{1} M\right|^{2}\left|F_{2} M\right|^{2}=\frac{1+s^{2}}{\left(1-s^{2}\right)^{2}}|O M|^{2}+\frac{s^{2}}{\left(1-s^{2}\right)^{4}},
$$

where $O=(0,0)$ and the points $F_{1}, F_{2}$ are the focuses

$$
F_{1}\left(\frac{-\sqrt{s}}{1-s^{2}}, 0\right), \quad F_{2}\left(\frac{\sqrt{s}}{1-s^{2}}, 0\right)
$$

see Figure 3.

We say that a closed curve $\gamma$ is convex when it is boundary of a convex bounded domain. Otherwise, we say that the curve $\gamma$ is concave. 


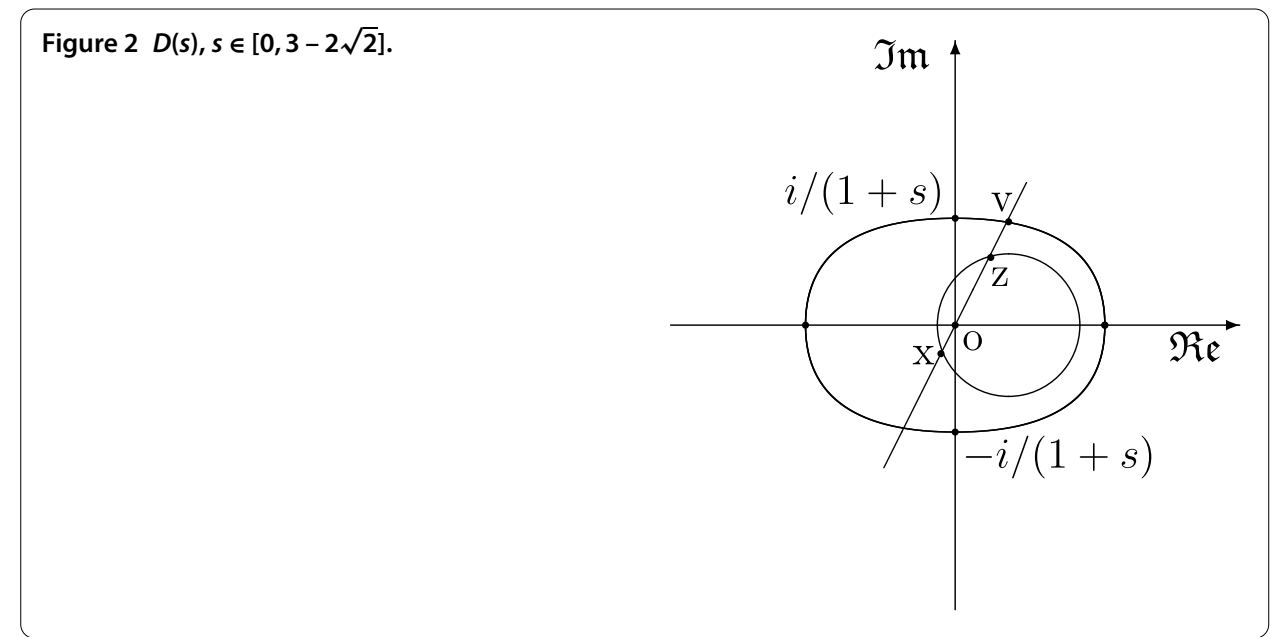

Figure $3 D(s), s \in(3-2 \sqrt{2}, 1)$.

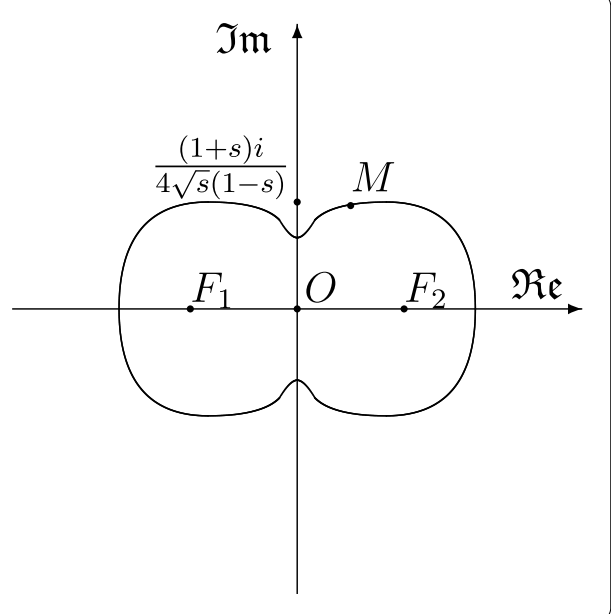

Lemma 3.2 Suppose that $F_{s}$ is given by (2.1). If $0 \leq s \leq 3-2 \sqrt{2} \approx 0.1715$, then the curve $F_{s}\left(e^{i \varphi}\right), \varphi \in[0,2 \pi)$ is convex and

$$
\max \left\{\mathfrak{I m}\left\{F_{s}\left(e^{i \varphi}\right)\right\}: \varphi \in[0,2 \pi)\right\}
$$

is attained at one point only. If $s \in(3-2 \sqrt{2}, 1)$, then the curve $F_{s}\left(e^{i \varphi}\right), \varphi \in[0,2 \pi)$ is concave and (3.10) is attained twice. Moreover, in both cases this curve is symmetric with respect to both axes.

Proof If $s=0$, then the curve $F_{s}\left(e^{i \varphi}\right), \varphi \in[0,2 \pi)$ becomes a circle and it is clear that (3.10) is attained one time only. Suppose, in the sequel, that $s \in(0,1)$. From (2.2) and (2.3) we have

$$
\mathfrak{R e}\left\{\frac{e^{i \varphi} F_{s}^{\prime \prime}\left(e^{i \varphi}\right)}{F_{s}^{\prime}\left(e^{i \varphi}\right)}+1\right\}=\frac{1-s^{4}+6 s\left(1-s^{2}\right) \cos \varphi}{\left(1+s^{2}\right)^{2}-4 s^{2} \cos ^{2} \varphi} .
$$


The denominator is positive, hence for the convexity of $F_{s}\left(e^{i \varphi}\right)$ we need the nominator to be positive. Since $s^{2}-6 s+1>0$ for $0 \leq s \leq 3-2 \sqrt{2}$, then the nominator is positive because

$$
\begin{aligned}
1-s^{4}+6 s\left(1-s^{2}\right) \cos \varphi & \geq 1-s^{4}-6 s\left(1-s^{2}\right) \\
& =\left(1-s^{2}\right)\left(s^{2}-6 s+1\right) .
\end{aligned}
$$

From (3.5) we have

$$
\mathfrak{I m}\left\{F_{s}\left(e^{i \varphi}\right)\right\}=\frac{(1+s) \sin \varphi}{1+s^{2}-2 s \cos 2 \varphi}:=\frac{(1+s) x}{1+s^{2}-2 s\left(1-2 x^{2}\right)}:=f(x)
$$

Then $f^{\prime}(x)=0$ if and only if $x^{2}=(1-s)^{2} /(4 s)$. That is possible when $(1-s)^{2} /(4 s) \leq 1$ because $x=\sin \varphi$. Therefore, using the elementary considerations, we can find that if $s \in(3-2 \sqrt{2}, 1)$, then the curve $F_{s}\left(e^{i \varphi}\right), \varphi \in[0,2 \pi)$ is concave and (3.10) is attained twice, when $\sin \varphi=(1-s) /(2 \sqrt{s})$. In this case,

$$
\max \left\{\mathfrak{I m}\left\{F_{s}\left(e^{i \varphi}\right)\right\}: \varphi \in[0,2 \pi)\right\}=\frac{1+s}{4 \sqrt{s}(1-s)}
$$

see Figure 3. Moreover, if $s \in[0,3-2 \sqrt{2}]$, then (3.10) is attained at one point only such that

$$
\max \left\{\mathfrak{I m}\left\{F_{s}\left(e^{i \varphi}\right)\right\}: \varphi \in[0,2 \pi)\right\}=\mathfrak{I m}\left\{F_{s}\left(e^{i \pi / 2}\right)\right\}=\frac{1}{1+s}
$$

see Figures 2 and 3.

Corollary 3.3 If $0 \leq s \leq 3-2 \sqrt{2} \approx 0.1715$, then $F_{s} \in \mathcal{K}$.

By Lemma 2.1 the functions $F_{s}, s \in[0,1]$, are starlike univalent, hence by the geometric interpretation (1.2) of a subordination under univalent functions, we obtain from Theorem 2.3 the following corollary.

Corollary 3.4 If $0 \leq s \leq 6-\sqrt{33}$ and $f \in \mathcal{H}$, then

$$
f(\Delta) \subset D(s) \quad \Longrightarrow \quad L[f](\Delta) \subset L\left[F_{s}\right](\Delta) .
$$

Theorem 3.5 Assume that $0<s_{i} \leq 3-2 \sqrt{2} \approx 0.1715, i=1,2$, and that the functions $g_{1}$ and $g_{2}$ are in the class $\mathcal{H}$ with $g_{1}(0)=g_{2}(0)=0$. If

$$
g_{1}(\Delta) \subset D\left(s_{1}\right) \text { and } g_{2}(\Delta) \subset D\left(s_{2}\right)
$$

then

$$
\left(g_{1} * g_{2}\right)(\Delta) \subset D\left(s_{1} s_{2}\right) .
$$

Proof Using Lemma 3.1, we may rewrite (3.12) as

$$
g_{1}(\Delta) \subset F_{s_{1}}(\Delta) \text { and } \quad g_{2}(\Delta) \subset F_{s_{2}}(\Delta) .
$$


By Corollary 3.3, $F_{s}$ is convex univalent in $\Delta$ for $s \in[0,3-2 \sqrt{2}]$, so $F_{s_{1}}, F_{s_{2}}$ are convex univalent in $\Delta$. From (1.2) and from (3.14), we obtain

$$
g_{1} \prec F_{s_{1}} \quad \text { and } \quad g_{2} \prec F_{s_{2}} \text {. }
$$

By Theorem D, we get from (3.15)

$$
g_{1} * g_{2} \prec F_{s_{1}} * F_{s_{2}} .
$$

But

$$
F_{s_{1}}(z) * F_{s_{2}}(z)=\frac{z}{1-s_{1} z^{2}} * \frac{z}{1-s_{2} z^{2}}=\frac{z}{1-s_{1} s_{2} z^{2}}=F_{s_{1} s_{2}}(z)
$$

and hence by Lemma 3.1 the subordination (3.16) is equivalent to (3.13).

Theorem 3.6 Assume that $0<s \leq 3-2 \sqrt{2} \approx 0.1715$ and that the functions $g_{1}$ and $g_{2}$ are in the class $\mathcal{H}$ with $g_{1}(0)=g_{2}(0)=0$. Let $\mathfrak{K}(c, R)$ be the disc with center $c \in \mathbb{R}$ and radius $R$ with $|c|<R$. If

$$
g_{1}(\Delta) \subset D(s) \text { and } g_{2}(\Delta) \subset \mathfrak{K}(c, R) \text {, }
$$

then

$$
\left(g_{1} * g_{2}\right)(\Delta) \subset \frac{R^{2}-c^{2}}{R} D\left(\frac{c s^{2}}{R^{2}}\right) .
$$

Proof Using Lemma 3.1, we may rewrite (3.17) as

$$
g_{1}(\Delta) \subset F_{s_{1}}(\Delta) \quad \text { and } \quad g_{2}(\Delta) \subset H_{c, R}(\Delta)
$$

where

$$
H_{c, R}(z)=\frac{\left(R^{2}-c^{2}\right) z}{R-c z} \quad(z \in \Delta)
$$

maps $\Delta$ onto $\mathfrak{K}(c, R)$. By Corollary $3.3, F_{s}$ is convex univalent in $\Delta$, also $H_{c, R}$ is convex univalent in $\Delta$. Therefore, by Theorem $\mathrm{D}$ we obtain

$$
\begin{aligned}
\left(g_{1} * g_{2}\right)(z) & \prec \frac{z}{1-s z^{2}} * \frac{\left(R^{2}-c^{2}\right) z}{R-c z} \\
& =\frac{R^{2}-c^{2}}{R} \frac{z}{1-s c^{2} z^{2} / R^{2}} \\
& =\frac{R^{2}-c^{2}}{R} F_{s c^{2} / R^{2}}(z) .
\end{aligned}
$$

The function $F_{s c^{2} / R^{2}}$ is univalent as the convolution of a convex univalent function, so by (1.2) we obtain (3.18). 
Theorem 3.7 Assume that $0<s \leq 3-2 \sqrt{2} \approx 0.1715$ and that the functions $g_{1}$ and $g_{2}$ are in the class $\mathcal{H}$ with $g_{1}(0)=g_{2}(0)=0$. Let $\mathfrak{L}(\varphi, d)=\left\{z \in \mathbb{C}: \mathfrak{R e}\left\{z e^{-i \varphi}\right\}>-d\right\}, \varphi \in \mathbb{R}, d>0$, be the half-plane. If

$$
g_{1}(\Delta) \subset D(s) \text { and } g_{2}(\Delta) \subset \mathfrak{L}(\varphi, d) \text {, }
$$

then

$$
\left(g_{1} * g_{2}\right)(\Delta) \subset 2 d e^{-i \varphi} D(s)
$$

Proof Using Lemma 3.1, we may rewrite (3.20) as

$$
g_{1}(\Delta) \subset F_{s_{1}}(\Delta) \text { and } g_{2}(\Delta) \subset G_{\varphi, d}(\Delta),
$$

where

$$
G_{\varphi, d}(z)=\frac{2 d e^{-i \varphi} z}{1-z} \quad(z \in \Delta)
$$

maps $\Delta$ onto $\mathfrak{L}(\varphi, d)$. By Corollary 3.3, $F_{s}$ is convex univalent in $\Delta$, also $G_{\varphi, d}$ is convex univalent in $\Delta$. Therefore, by Theorem $\mathrm{D}$ we obtain

$$
\begin{aligned}
\left(g_{1} * g_{2}\right)(z) & \prec \frac{z}{1-s z^{2}} * \frac{2 d e^{-i \varphi} z}{1-z} \\
& =\frac{2 d e^{-i \varphi} z}{1-s z^{2}}=2 d e^{-i \varphi} F_{s}(z) .
\end{aligned}
$$

Hence by (1.2) we obtain (3.21).

\section{Competing interests}

The authors declare that they have no competing interests.

\section{Authors' contributions}

All authors read and approved the final manuscript.

Received: 20 April 2013 Accepted: 15 August 2013 Published: 3 September 2013

\section{References}

1. Ruscheweyh, S, Sheil-Small, T: Hadamard product of schlicht functions and the Polya-Schoenberg conjecture. Comment. Math. Helv. 48, 119-135 (1973)

2. Stankiewicz, J, Stankiewicz, Z: Some applications of the Hadamard convolution in the theory of functions. Ann. Univ. Mariae Curie-Sklodowska, Sect. A 40, 251-265 (1986)

3. Piejko, K, Stankiewicz, J: Convolution of functions with free normalization. Demonstr. Math. 34(1), 69-76 (2001)

4. Miller, SS, Mocanu, PT, Reade, MO: Subordination preserving integral operators. Trans. Am. Math. Soc. 283(2), 605-615 (1984)

5. Libera, RJ: Some classes of regular univalent functions. Proc. Am. Math. Soc. 16, 755-758 (1965)

6. Hallenbeck, DI, Ruscheweyh, S: Subordination by convex functions. Proc. Am. Math. Soc. 52, 191-195 (1975)

7. Ruscheweyh, S, Stankiewicz, J: Subordination under convex univalent function. Bull. Pol. Acad. Sci., Math. 33, 499-502 (1985)

8. Ruscheweyh, S: Convolutions in Geometric Function Theory. Sem. Math. Sup., vol. 83. Presses University Montreal, Montreal (1982)

9. Wang, Z-G, Sun, Y, Xu, N: Some properties of certain meromorphic close-to-convex functions. Appl. Math. Lett. 25(3), 454-460 (2012)

10. Deniz, E, Răducanu, D, Orhan, H: On the univalence of an integral operator defined by Hadamard product. Appl. Math. Lett. 25(2), 179-184 (2012)

11. Faisal, I, Darus, M: A study of a special family of analytic functions at infinity. Appl. Math. Lett. 25(3), 654-657 (2012) 
12. El-Ashwah, RM, Aouf, MK: The Hadamard product of meromorphic univalent functions defined by using convolution. Appl. Math. Lett. 24(12), 2153-2157 (2011)

13. Sarkar, N, Goswami, P, Bulboacă, T: Subclasses of spirallike multivalent functions. Math. Comput. Model. 54(11-12), 3189-3196 (2011)

14. Rogosinski, W: On the coefficients of subordinate functions. Proc. Lond. Math. Soc. 48(2), 48-82 (1943)

15. Booth, J: A Treatise on Some New Geometrical Methods, vol. I. Longmans, Green, London (1873)

16. Booth, J: A Treatise on Some New Geometrical Methods, vol. II. Longmans, Green, London (1877)

doi:10.1186/1029-242X-2013-420

Cite this article as: Piejko and Sokół: Hadamard product of analytic functions and some special regions and curves. Journal of Inequalities and Applications 2013 2013:420.

\section{Submit your manuscript to a SpringerOpen ${ }^{\odot}$} journal and benefit from:

- Convenient online submission

Rigorous peer review

- Immediate publication on acceptance

- Open access: articles freely available online

- High visibility within the field

- Retaining the copyright to your article 